From a theoretical point of view we have to face the following problems:

I. The old problem of understanding the basic hydrodynamics of the outer stellar convection zones (which, as we believe, are also at least the energy sources of the observed oscillations and other photospheric phenomena) is still very far from being solved, despite large computational efforts in the last three years.

2. A fundamental problem is related to the transition from essentially convective modes in deeper layers to oscillatory or wave modes in higher layers of the photosphere. Some tentative calculations indicate a strong overshooting of purely convective modes into the higher layers of the solar atmosphere. Earlier estimates of acoustic noise generated in the convection zone lead to fairly small amplitudes in the upper photospheric layers. Why do we then observe mainly oscillatory motion in the upper photosphere?

3. Why do the observed oscillations have such a fairly narrow frequency spectrum? How does the spectrum change with height in the photosphere?

4. Can we understand the fact that the super-granulation is observed essentially only in the chromosphere if it is an overshooting phenomenon of purely convective modes?

5. If the existence of waves and oscillations in the solar atmosphere is taken for granted, how do these waves propagate in a stratified atmosphere with a temperature minimum?

6. Under which circumstances can these waves propagate into the corona?

It is hoped that this Joint Discussion will lead to the suggestion of some new approaches to these difficult and urgent problems.

\title{
2. OBSERVATIONAL KNOWLEDGE OF MOTIONS IN THE SOLAR ATMOSPHERE
}

\section{R. Michard}

(Observatoire de Paris, Meudon)

Motions in the normal solar atmosphere can be studied first by their effects on average strengths and profiles of Fraunhofer lines ('astrophysical turbulence'). Sufficiently thick velocity elements, if larger than about $\mathrm{I}^{\prime \prime}$, can be detected individually and their properties described as a function of time and space. Further, a moving atmosphere cannot be homogeneous and conversely an atmosphere with local fluctuations of $T$ and $p$ cannot be at rest: the study of purely photometric fluctuations, such as the photospheric granulation, is thus intimately connected to the study of the mechanics of the solar atmosphere.

\section{Astrophysical Turbulence in the Solar Atmosphere}

The existence of 'astrophysical turbulence' in the solar atmosphere can be derived first by the same methods which apply in the case of stars:

I. The study of line-strengths by the curve of growth shows that the 'micro-turbulence' is about $\xi=\mathrm{I} \cdot 2 \mathrm{~km} / \mathrm{sec}$.

2. The study of Fraunhofer line profiles has been made for lines of different strengths, by many workers using various methods of analysis. Of great importance is the extension of this type of work to the chromosphere, started by Redman and Suemoto from spectra of the $195^{2}$ eclipse, with further progresses made by Suemoto from material obtained at the 1958 eclipse. 
Very serious difficulties are associated with any attempts to derive 'turbulence' from line profiles, and still larger difficulties with the approximate methods of analysis used by most workers. A sufficiently reliable measurement of profiles is also not too easy. For these reasons there are large discrepancies in the results obtained so far: however, they can be summarized as follows:

A total 'turbulent velocity' $\xi$ of about $2 \mathrm{~km} / \mathrm{sec}$ exists in the upper photosphere where most metallic lines are formed.

This velocity may be anisotropic, with horizontal motions larger than vertical ones.

The turbulent velocity increases in the chromosphere up to values of more than $10 \mathrm{~km} / \mathrm{sec}$. These very large velocities represent the random motions of 'spicules', a particular structure which occupies about one per cent of the chromosphere.

The variation of $\xi$ towards deep photospheric layers is not clear; it is probably increasing with depth. Accordingly there is a suggestion that the $\xi$ values pass through a minimum in the photosphere.

The comparison of $\xi$ values from the curve of growth and from line profiles suggests that both 'micro-' and 'macro-turbulent' solar motions exist, that is both small transparent 'elements' and large, optically thick, structures contribute to the velocities. The question of the relative importance of both types of turbulence is still controversial.

\section{Local Doppler Shifts of Fraunhofer Lines}

In 1950, Richardson and Schwarzschild published the first results on solar turbulent motions detected from local Doppler shifts or 'wiggles' of the Fraunhofer lines. They found that these wiggles had an r.m.s. of $0.26 \mathrm{~km} / \mathrm{sec}(\xi=\sqrt{2}$ r.m.s. $=0.37)$ and a geometrical scale similar to the scale of the solar granulation or larger; velocities were correlated with continuum brightness, with upwards motions generally associated with bright features, the coefficient of correlation being only 0.30 . Further work in the ro following years involved the measurements of wiggles on many lines of selected spectrograms, both at the centre of the disk and at near limb positions.

The main results may be summarized as follows:

At the centre of the disk, where we observe vertical velocities, the parameter $\xi$ increases with line-strength, from values of about $0.25 \mathrm{~km} / \mathrm{sec}$ for average lines (Rowland intensity 2) to $2 \mathrm{~km} / \mathrm{sec}$ or more for $\mathrm{H} \alpha$ or the core $\mathrm{K}_{3}$ of the $\mathrm{K}$-line. It also shows dependence for a given line, from the distance $\Delta \lambda$ of the line centre at which the shift is measured. For lines of a given strength, $\xi$ varies with the spectral range being for instance distinctly larger in the near UV. All these variations are in the sense that the macroscopic motions increases with height in the solar atmosphere. However, there is a rather marginal indication that for very faint lines, or deep layers, $\xi$ also increases.

The centre-limb variation of $\xi$ is dependent upon line-strength, and probably dominated by anisotropy of the velocity-field. There is good indication that we observe the superposition of a mainly vertical velocity-field with marked height increase, and of a mainly horizontal field with no large height variation.

Two lines on a given spectrogram show correlated wiggles, but the coefficient of correlation decreases rapidly when we consider two lines of increasingly different strength (or level of formation). This rules out a columnar structure of the velocity-field.

The coefficient of correlation of velocities with the continuum granulation is generally in the range $0.2-0.4$. It shows, of course, rather large changes from one spectrogram to the other, as a result of sampling fluctuations. These changes are, however, larger than is expected from 
available statistical criteria, and this led Edmonds to suggest that they may represent real changes in the properties of solar inhomogeneities from one place to another on the Sun.

\section{Recent Observational Advances}

In 1960 and I961, the study of macroscopic motions took a new turn as a result of observational developments which permitted to obtain much larger amounts of data, and to study the time-variations of solar structure.

Leighton at Mount Wilson developed a beautiful technique: he used a spectroheliograph to make simultaneously two monochromatic pictures of the Sun, with two pass-bands symetrical with respect to the centre of a line, one in the violet slope; one in the red slope of the line profile. A positive contact print at contrast unity of one of the pictures, is superposed on the negative of the other: in this composite picture, the local variations of density arise essentially from the local Doppler shifts of the line, while brightness fluctuations, both solar and instrumental, are cancelled. Such a 'Doppler plate' gives a vivid picture of the distribution of motion on the Sun. The time needed to take a spectroheliogram with a narrow pass-band is a few minutes: but making two consecutive plates with opposite directions of scanning, one obtains a continuously changing time interval between the two exposures from one edge to the other of the picture. In this way Leighton could observe time variations, and he discovered the vertical oscillations of the solar atmosphere which will be discussed later.

In Sacramento Peak, Evans was able to obtain time sequences of high resolution spectrograms, with good image quality for periods of nearly half an hour; the stability of the solar image on the slit was controlled by a photo-electric guiding. Such sequences allow the simultaneous study of time variations for many spectral features, such as shifts of a number of spectral lines, continuum granulation, line depths and profiles. The correlations and phase differences between these can be analysed.

\section{The Supergranulation}

The examination of one of Leighton's 'Doppler plates' shows at first glance a very important difference in the spatial structure of the field of vertical velocities (observed near the centre of the disk) and the field of horizontal velocities (observed near the limb). The pattern of vertical velocities is an apparently random one with characteristic cell sizes in the range $1000-3000 \mathrm{~km}$. On the other hand, the horizontal motions are predominantly found in large cells of about $30000 \mathrm{~km}$ which show a typical inner structure: the part of the cell towards the limb of the Sun shows velocities of recession and the reverse for the part towards the centre of the disk. This suggests a radial flow from the centre of each cell towards its periphery.

This rather organized pattern of horizontal motions has been called by Leighton 'supergranulation', suggesting that it is a convective phenomenon as the small scale photospheric granulation is supposed to be. The supergranulation is a slowly evolving phenomenon, the pattern being easily recognized after a few hours. The question of the vertical extension of the corresponding flow is not yet entirely clear. It seems that it is confined to the photosphere, or more probably masked by faster motions of a different type in the chromosphere.

On the other hand, Simon showed that the supergranulation is connected with the coarse network of calcium spectroheliograms. Bright calcium features occupy the spaces in between supergranulation cells. It is further known from many observations that bright calcium flocculi are associated with magnetic fields of the order of Io gauss or more. Finally, they are the roots of bushes of 'spicules', these being short streamers of relatively dense matter ascending in the corona with velocities of the order of $20-40 \mathrm{~km} / \mathrm{sec}$ : this is shown beautifully by high quality $\mathrm{H} \propto$ spectroheliograms from Mount Wilson. 
The flow of the supergranulation is superposed upon a system of mainly vertical oscillatory motions which will be our main subject later. It is an interesting question to ask whether or not there is a correlation of the supergranulation cells with local anomalies in the vertical velocities, delineating the places were the horizontal flow lines bend upwards or downwards. A positive answer to this question is given in recent work by Simon.

\section{Vertical Velocity Field}

The most detailed results about solar vertical motions have been obtained from Sacramento Peak time sequences of spectrograms of the centre of the disk. The properties of vertical velocities have been investigated for some 12 lines, which scan completely the possible range of levels of formation from the deep photospheric line $5052 \cdot I 6$ of $\mathrm{CI}$ to chromospheric lines like $\mathrm{H} \alpha$ and $\mathrm{K}$.

As first reported by Leighton in 1960 , the motions from average lines are dominated by oscillations with a privileged period of $295 \mathrm{sec}$. But the actual velocity-field ( $V$-field) is of course more complex than a system of mono-frequency waves, and should be described by its Fourier power-spectrum $G(v)$. Similarly the correlations between two different fluctuating phenomena will be described by their 'coherence' and 'phase' as a function of frequency.

These functions are obtained by standard methods of numerical analysis and provide an objective description of the random-like functions under study. However, their accuracy is limited by the finite extent of the amount of data which is used to calculate them. If the length of time covered by the available data is $\tau$, the Fourier frequency spectrum is obtained with an 'apparatus function' of total half-width $\mathrm{I} / \tau$. The finite dimensions of the samples of data also introduce limitations in the significance of the results, due to sampling errors.

We next describe the power spectra of the $V$-field in 3 medium to very faint lines. The power is mainly concentrated into a 'resonance range' with mean period $300 \mathrm{sec}$. With increasing height the resonance sharpens and the peak is shifted towards higher frequencies. There is also significant power in low frequencies $\left(\nu<10^{-3} \mathrm{~Hz}\right)$ with amount increasing with depth. In the frequency power spectrum of photospheric granulation measured from the same sequence of spectrograms, the power is concentrated in low frequencies and decreases towards high frequencies, with a kind of hump on the curve, in the resonance range of velocities.

From this comparison, we are tempted to conclude that the low-frequency end of $V$-power spectra represents a 'convective' component in the $V$-field. This component increases with depth as it should. The presence of a faint oscillatory component in the granulation is also possible.

When we examine power spectra for stronger lines, we recognize the resonance range, still drifting towards higher frequencies with increasing height. The low frequency 'convective' part goes through a minimum for the $b_{2}$ line of $\mathrm{MgI}$, then it increases again becoming strong for chromospheric lines. However, it can be shown that the low-frequency $V$-fields of deep photospheric lines and of chromospheric lines are entirely distinct phenomena. The chromospheric persistent $V$-field is probably linked with the coarse mottling of spectroheliograms but this has not been proved.

In the power-spectra of strong lines, from $b_{2}$ to $H \alpha$ we recognize also a 'high frequency tail' whose relative strength increases with height in the atmosphere.

Although the measured velocities are some average along the line of sight, it is tempting to try to associate a 'level' to each of our lines in order to examine the transfer of mechanical energy in the atmosphere. This is tentatively done in Table $\mathbf{I}$.

From the Table it is seen that the kinetic energy $\rho \xi^{2}$ per unit volume in the form of macroscopic motions begins to decrease sharply around the o $\mathrm{km}$ level, that is near the temperature 


\begin{tabular}{|c|c|c|c|c|c|c|}
\hline \multirow{2}{*}{ Line } & \multirow{2}{*}{$\begin{array}{c}\text { 'Height' } \\
\text { (km) }\end{array}$} & \multirow{2}{*}{$\begin{array}{l}\text { Density } \\
\rho \times 10^{9}\end{array}$} & \multirow{2}{*}{$\xi^{2} \times 10^{-10}$} & \multirow{2}{*}{$\rho \xi^{2}$} & \multicolumn{2}{|c|}{$\rho \xi^{2}$} \\
\hline & & & & & $\begin{array}{c}\text { In resonance } \\
\nu=2 \cdot 75-4.25\end{array}$ & $\begin{array}{c}\text { In HF tail } \\
(\nu=5.75-8.25\end{array}$ \\
\hline $5052 \mathrm{CI}_{\mathrm{I}}$ & -320 & 253 & 0.057 & I 45 & 66 & 4 \\
\hline $85 I_{4} \mathrm{Fe}$ & -175 & I 20 & 0.109 & I 30 & 74 & 6 \\
\hline $5172 \mathrm{Mg} \mathrm{I}$ & +100 & II & 0.36 & 40 & 22 & 3.5 \\
\hline $8498 \mathrm{Ca}$ II & +400 & I & 0.39 & 4 & $\mathrm{I} \cdot 4$ & 0.65 \\
\hline $8542 \mathrm{Ca}$ II & +700 & 0.3 & $I \cdot 0$ & $2 \cdot 9$ & 0.75 & 0.75 \\
\hline $\mathbf{H}_{\alpha}$ & $(2000)$ & 0.01 & 4.0 & 0.2 & 0.07 & 0.08 \\
\hline
\end{tabular}

minimum of the atmosphere. This attenuation is much more marked for the resonance range than for higher frequencies. Of course this refers only to 'macroscopic' motions, that is to a very small fraction of the total kinetic energy at photospheric levels, a fraction which is of the order of 5 per cent.

We come now to the discusssion of phase transfer properties of the $V$-field. This question was studied first by Evans and Michard who compared the run of velocities in two lines of widely different strengths in a sample of 40 'prominent oscillations'. In practically all cases, Doppler shifts in the high level line lagged behind Doppler shifts in the deeper line. The lag was 8 sec. in the average and seemed much smaller than it should have been for sound waves. There was a relation between periods and time-lags for individual oscillations, the oscillations with the longest periods showing the smallest timelags. On the other hand, it appeared that during the lifetime of a single oscillation, the time-lag decreased from a maximum value at the real (or apparent) beginning of the perturbation, to zero after three half-periods. This suggests that an oscillation starts as a progressive wave, possibly at sound velocity, then rapidly decays into a standing oscillation.

It may be mentioned here that the average decay time of oscillations is of the order of Io minutes from the auto-correlation curves. They seem to disappear by interference with new perturbations rather than by actual damping. A few individual oscillations lasting for three periods, or ${ }_{5}$ minutes, have been observed; the amplitude often remains constant or even increases during the life of an oscillation, and its disappearance is generally abrupt.

The phase difference of the complete $V$-fields of two lines can also be calculated as a function of frequency by statistical analysis. All results for many pairs of lines, show that there is some progressive motion in the resonance range of the $V$-field, with phase propagation velocities much larger in the mean than the speed of sound. Larger phase differences are found for pairs of lines that are the more different in strength and level of formations.

Outside the resonance range, coherence and phase calculations are less significant because the spectral power is too small. The phase-lag between two lines is generally found to increase with frequency after the resonance peak, as can be expected theoretically, when we go from resonance standing oscillations towards ordinary sound waves. The results suggest that frequencies of the order of twice the resonance frequency are actually sound waves. A notable exception to this rule was found in comparing the chromospheric high frequency tails observed in 
Doppler shifts of the two lines 8498 and 8542 of CaIr: these were in phase which makes somewhat puzzling the explanation of this part of the kinetic spectra of the solar atmosphere. As regards the low frequency 'convective' part of power spectra no significant results about phases could be obtained.

We shall now discuss the statistical relations of $V$-fields with the continuum granulation, considering first what happens in the resonance range of the $V$-field. A qualitative examination of one good Sacramento Peak sequence, suggested that the appearance of a bright granule tends to start an oscillation, beginning with upwards motion. This result should be checked from a still better and larger material. However, it is compatible with the results from statistical analysis.

We next describe the coherence and phase functions between velocities in a faint line and granulation, from one particularly long and good sequence of spectrograms. In the range of resonance oscillations, we have a significant coherence of 0.5 and the phase lag between positive upwards velocities and positive bright features is about $40^{\circ}$ or roughly 50 seconds for this range of periods. At low frequencies we have also a large coherence (more than 0.5 ) between the two phenomena, and a phase in the sense opposite to the one in the resonance range; in between there is a region where the coherence decreases abruptly while the phase changes suddenly.

I must confess that I am not yet very clear about the significance of this and similar results, except that probably they confirm the existence of two components in the $V$-field, a 'convective' and an oscillatory one, both being somehow connected with the granulation and convection in the deep photosphere. The analysis of a number of good films is needed to disentangle what is definitely significant in these statistical analysis.

Very important information on thermodynamical fluctuations associated with motions in the solar atmosphere may finally be derived from the study of brightness fluctuations at the centre, or in the profiles, of Fraunhofer lines. On Sacramento Peak films have been studied the deeply formed line of carbon at $5052 \cdot 16$ and also the upper photospheric lines $5 \mathrm{I} 7 \mathrm{I} \cdot 6$ of FeI and $b_{2}$ of $\mathrm{Mg}$ I. Orrall and Jensen described variations in the wings of the $\mathrm{K}$ line. Data on brightness fluctuations in the profile of a few lines were also reported by Noyes and Leighton.

The results differ widely according to the line chosen. For instance, brightness fluctuations in the centre of the faint carbon line have a power spectrum nearly identical with the one of continuum granulation, and are well correlated with these, though with a definite phase lag.

On the other hand, fluctuation in a strong line, like 5I7I Fe I show a time power spectrum made up of two parts: oscillations in the same range of periods as the resonance oscillations in velocities, and a low frequency part. The brightness oscillations are nicely coherent with $V$ oscillations and nearly in quadrature with them. This part of the brightness fluctuations spectrum is accordingly a consequence of the variations $\Delta T$ and $\Delta p$ in oscillatory motions.

On the other hand the low frequency changes of brightness for strong lines are not correlated with the granulation and should possibly be related to the coarse mottling of spectroheliograms.

\section{Conclusion}

We have tried to summarize and classify the various types of inhomogeneities observed in the solar atmosphere in the accompanying Table 2.

For the sake of simplification, we may introduce the following further classification by probable physical origin.

I. Small-scale 'deep' convection: Granulation, low frequency photospheric $V$-field, low frequency faint lines profiles fluctuations $(1,2 a, 3 c)$.

II. Resonance phenomena: Oscillations in $V$-field, brightness oscillations in strong lines $\left(2 b, 3^{b}\right)$. 
Table 2

Summary of Observed Structures in the Normal Solar Atmosphere

\begin{tabular}{|c|c|c|c|c|c|c|c|}
\hline $\begin{array}{l}\text { Serial } \\
\text { no. }\end{array}$ & Name & $\begin{array}{l}\text { Observed } \\
\text { from }\end{array}$ & $\begin{array}{c}\text { Scale } \\
\text { space } \\
\text { spectrum }\end{array}$ & $\begin{array}{l}\text { Duration } \\
\text { time } \\
\text { spectrum }\end{array}$ & $\begin{array}{l}\text { Height } \\
\text { extent and } \\
\text { variations }\end{array}$ & $\begin{array}{l}\text { Corre- } \\
\text { lated } \\
\text { with }\end{array}$ & $\begin{array}{l}\text { Possible } \\
\text { physical } \\
\text { origin }\end{array}$ \\
\hline I & Granulation & $\Delta I_{\mathrm{c}}$ & $1700 \mathrm{~km}$, flat & $\begin{array}{l}\text { Io min, } \\
\text { decay type }\end{array}$ & Photosphere & 2 & $\begin{array}{l}\text { Convection } \\
\text { (oscillatory } \\
\text { modes in } \\
\text { part?) }\end{array}$ \\
\hline $2 a$ & $\begin{array}{l}\text { Low frequency } \\
\text { photospheric } \\
V \text {-field }\end{array}$ & $\int \Delta \lambda$, vertical & $\mathrm{I}-3000 \mathrm{~km}$ ? & $\begin{array}{l}>\text { Io min } \\
\text { decay type }\end{array}$ & $\begin{array}{c}\text { Deep photo- } \\
\text { sphere, } \xi \searrow\end{array}$ & I & Convection \\
\hline $2 b$ & Oscillations & $\Delta \lambda$, vertical & $\mathrm{I}-3000 \mathrm{~km}$ & $\begin{array}{l}\text { Band around } \\
t=300 \text { sec } \\
\text { dominates } \\
\text { macro. } \\
V \text {-field }\end{array}$ & $\begin{array}{l}\text { Photosphere, } \\
\text { low chromo- } \\
\text { sphere, } \xi \nearrow\end{array}$ & $1,4 b$ & $\begin{array}{l}\text { Resonance } \\
\text { oscillations }\end{array}$ \\
\hline $2 c$ & $\begin{array}{l}\text { High-frequency } \\
\text { tail of } V \text {-field }\end{array}$ & $\Delta \lambda$, vertical & Small scale & $\begin{array}{l}\text { Periods in } \\
\text { range } \\
200-100 \mathrm{sec}\end{array}$ & $\begin{array}{l}\text { Low chromo- } \\
\text { sphere, } \xi \nearrow\end{array}$ & $?$ & $?$ \\
\hline $2 d$ & $\begin{array}{l}\text { Low frequency } \\
\text { chromospheric } \\
V \text {-field }\end{array}$ & $\Delta \lambda$, vertical & $?$ & Decay type & $\begin{array}{l}\text { Low chromo- } \\
\text { sphere, } \xi \nearrow\end{array}$ & $?$ & $\begin{array}{l}\text { Possibly } \\
\text { related to } 3 a\end{array}$ \\
\hline $3 a$ & $\begin{array}{l}\text { Coarse mottling } \\
\text { and network }\end{array}$ & $\begin{array}{l}\Delta I_{\lambda} \\
\text { Strong lines }\end{array}$ & $>10000 \mathrm{~km}$ & Hours & $\begin{array}{l}\text { Low chromo- } \\
\text { sphere }\end{array}$ & $\begin{array}{l}\text { Magn. } \\
\text { fields } \\
\text { and } 5\end{array}$ & $\begin{array}{l}\text { Convective } \\
\text { modes? }\end{array}$ \\
\hline$\left\{3^{b}\right.$ & $\begin{array}{l}\text { Brightness } \\
\text { oscilations }\end{array}$ & $\begin{array}{l}\Delta I_{\lambda} \\
\text { Strong, } \\
\text { average I }\end{array}$ & $\mathrm{I}-3000 \mathrm{~km}$ & $\begin{array}{l}\text { Band around } \\
t=300 \mathrm{sec}\end{array}$ & $\begin{array}{l}\text { Upper photo- } \\
\text { sphere, low } \\
\text { chromosphere }\end{array}$ & $2 b$ & $\begin{array}{l}\Delta T \text { and } \Delta p \text { of } \\
\text { resonance } \\
\text { oscillations }\end{array}$ \\
\hline$(3 c$ & $\begin{array}{l}\text { Low frequency } \\
\text { brightness } \\
\text { fluctuations }\end{array}$ & $\begin{array}{l}\Delta I_{\lambda}, \\
\text { average, } \\
\text { faint I }\end{array}$ & $\mathrm{I}-3000 \mathrm{~km}$ ? & $\begin{array}{l}\text { Io min, decay } \\
\text { type }\end{array}$ & $\begin{array}{l}\text { Deep photo- } \\
\text { sphere }\end{array}$ & I & Convection \\
\hline 4 & $\begin{array}{l}\text { 'Supergranu- } \\
\text { lation' }\end{array}$ & $\begin{array}{l}\Delta \lambda, \text { hori- } \\
\text { zontal }\end{array}$ & $>10000 \mathrm{~km}$ & Hours & Photosphere? & $3 a$ & Convection? \\
\hline 5 & Spicules & $\begin{array}{l}\Delta I_{\lambda} \\
\Delta \lambda \text { vertical }\end{array}$ & $\begin{array}{l}\text { rooo km } \\
\text { localized } \\
\text { in bushes }\end{array}$ & $5 \mathrm{~min}$ & $\begin{array}{l}\text { Upper } \\
\text { chromo- } \\
\text { sphere }\end{array}$ & $\begin{array}{l}\text { 3a? } \\
\text { Magn. } \\
\text { fields? }\end{array}$ & $\begin{array}{l}\text { Suppression } \\
\text { of oscil- } \\
\text { lations? }\end{array}$ \\
\hline 6 & Micro $V$-field & $\begin{array}{c}\text { Curve of } \\
\text { growth, } \\
\text { profiles }\end{array}$ & $<500 \mathrm{~km}$ & $?$ & $\begin{array}{l}\text { All atmo- } \\
\text { sphere } \xi \nearrow, \\
\text { dominates } \\
V \text {-field }\end{array}$ & $?$ & Sound waves? \\
\hline
\end{tabular}

III. Large-scale convection (?): Chromospheric coarse mottling, 'supergranulation', low frequency part of vertical chromospheric $V$-fields? $(3 a, 4 d$ ?).

IV. High frequency sound waves?: Microturbulence (6).

The spicules are left outside this classification because they are localized structures involving a very small part of the solar surface. The phenomena I disappear with increasing height in the photosphere; phenomena II occupy the photosphere and low chromosphere with maximum importance around the temperature minimum. Phenomena III are more or less recognizable through the photosphere and low chromosphere. 
As a conclusion we shall comment upon phenomena IV, the 'unseen mechanical energy' which certainly exists besides this part of the $V$-field detected by lines wiggles.

Unpublished estimates of the smoothing of the macro-turbulent $V$-field by insufficient resolving power, suggest that the true macroscopic motions $\xi^{2}$ are only a factor of 3 to 5 higher than observed ( $\mathrm{I} \cdot 7$ to 2.3 on $\xi$ ). Therefore the macroscopic motions remain smaller by a factor of 3 or more, than the turbulent velocities derived from the curve of growth and profiles. The motions involving optically thin volume elements are accordingly the essential part of the mechanical energy in the solar stmosphere.

According to Osterbrock, the acoustical noise spectrum from the deep convection zone might be peaked around $\nu_{0}=\mathrm{I} \cdot 2 \times 10^{-2} \mathrm{~Hz}(t=80 \mathrm{sec})$. Sound waves at this supposed peak and higher frequencies have a wavelength $\Lambda$ of $500 \mathrm{~km}$ and smaller and cannot give observable line shifts, because the thickness of line formation layers extends on a length which is a large fraction of $\Lambda$. Also we may expect the extent of the fronts of such short wave elements to be small (say of the order of $\Lambda$ ) and below the possibility of resolution. So we suggest that the microscopic $V$-field transported through the atmosphere (and mainly responsible for coronal heating) is high frequency, that is acoustical noise around $10^{-2} \mathrm{~Hz}$.

\title{
DISCUSSION
}

R. Michard (answering questions by Dr Giovanelli and Dr Rösch). Concerning the sketch summarizing the 'supergranulation', I completely agree that his picture is still tentative. In particular it is not proved that the pattern of downwards flow in $\mathrm{H} \alpha$ is related to the supergranulation.

F. Rösch. It is possible that changes in the brightness-velocity correlation for photospheric granules will occur when the image resolution is increased.

\section{THE SOLAR HYDROGEN CONVECTION ZONE AND ITS DIRECT INFLUENCE ON THE PHOTOSPHERE}

\author{
E. A. Spiegel \\ (Physics Department, New York University)
}

The diversity of phenomena in the solar atmosphere, which observers have been discovering at a dramatically accelerated pace in recent years, seems to defy all attempts at a general chacterization. Nevertheless, it seems clear that the energy implied by the observed inhomogeneities, velocity fields, and magnetic fields, is derived from the motions originating in the solar hydrogen convection zone. Thus, for a complete understanding of solar phenomena we certainly require not only a theory of convective motion, but also an understanding of the couplings among the various modes of energy which are observed or inferred. Neither of these is really available, in spite of some recent progress in the understanding of laboratory convection, and it is likely that the two problems can only be solved together. The upshot is that a gap exists between the present exciting picture provided by solar observers and the type of phenomenon that the theory of convection can even clarify qualitatively. In spite of this very real separation between the realms of observational and theoretical knowledge a discussion of the interaction between the convection zone and the photosphere may be useful at this time, if only to illustrate the present possibilities for theoretical interpretation. 